\title{
Principais abordagens fisioterapêuticas no tratamento de artrite reumatóide: uma revisão bibliográfica
}

\author{
Main physical therapy approaches in the treatment of rheumatoid arthritis: a literature review \\ Principales enfoques de fisioterapia en el tratamiento de la artritis reumatoide: revisión de la
}

literatura

Recebido: 18/09/2021 | Revisado: 23/09/2021 | Aceito: 24/09/2021 | Publicado: 25/09/2021

Luiz Paulo Sobral Pereira

ORCID: https://orcid.org/0000-0002-7769-1634

Clínica Ortofisio, Brasil

E-mail: luizpaulofisioterapeuta@gmail.com

Mirla da Silva Maia

ORCID: https://orcid.org/0000-0001-9355-6582

Clínica Ortofisio, Brasil

E-mail:mirladsm@gmail.com

\begin{abstract}
Resumo
A artrite reumatóide é uma patologia inflamatória e autoimune que atinge os tecidos articulares levando o organismo a desenvolver deformidades e limitações de movimento prejudicando as atividades de vida diária e laborais, porém ainda não há um consenso quanto a aplicação das técnicas fisioterapêuticas em indivíduos com artrite reumatóide. Com isso, o estudo buscou informações sobre as principais abordagens fisioterapêuticos a serem empregadas no tratamento desta patologia que ajudam a controlar os sinais e sintomas da doença. De acordo com a pesquisa feita, os métodos de hidroterapia, massagem terapêutica, laserterapia e exercícios físicos mostraram sua eficácia no quadro de saúde de indivíduos com a patologia, porém não se obteve consenso quanto aos parâmetros dos métodos. Portanto, o estudo demonstrou-se relevante para a análise da efetividade das abordagens fisioterapêuticas em indivíduos com artrite reumatóide.
\end{abstract}

Palavras-chave: Artrite reumatóide; Exercício físico; Fisioterapia; Tratamento.

\begin{abstract}
Rheumatoid arthritis is an inflammatory and autoimmune pathology that affects the joint tissues, leading the body to develop deformities and movement limitations, impairing daily life and work activities, but there is still no consensus on the application of physical therapy techniques in individuals with rheumatoid arthritis . Thus, the study sought information about the main physical therapy approaches to be used in the treatment of this pathology, which help to control the signs and symptoms of the disease. According to the research carried out, the methods of hydrotherapy, therapeutic massage, laser therapy and physical exercise showed their effectiveness in the health status of individuals with the pathology, but there was no consensus on the parameters of the methods. Therefore, the study proved to be relevant for the analysis of the effectiveness of physical therapy approaches in individuals with rheumatoid arthritis.

Keywords: Rheumatoid arthritis; Physical exercise; Physiotherapy; Treatment.
\end{abstract}

\section{Resumen}

La artritis reumatoide es una patología inflamatoria y autoinmune que afecta los tejidos articulares, llevando al organismo a desarrollar deformidades y limitaciones de movimiento, perjudicando la vida diaria y las actividades laborales, pero aún no existe consenso sobre la aplicación de técnicas de fisioterapia en personas con artritis reumatoide. Así, el estudio buscó información sobre los principales enfoques de fisioterapia a utilizar en el tratamiento de esta patología, que ayuden a controlar los signos y síntomas de la enfermedad. Según la investigación realizada, los métodos de hidroterapia, masaje terapéutico, terapia con láser y ejercicio físico mostraron su efectividad en el estado de salud de las personas con la patología, pero no hubo consenso sobre los parámetros de los métodos. Por lo tanto, el estudio demostró ser relevante para el análisis de la efectividad de los enfoques de fisioterapia en personas con artritis reumatoide.

Palabras clave: Artritis reumatoide; Ejercicio físico; Fisioterapia; Tratamiento. 


\section{Introdução}

A artrite reumatóide (A.R.) pode ser definida com uma patologia crônica, inflamatória e autoimune, na qual o sistema imunológico do organismo ataca os tecidos que envolvem as articulações, podendo levar ao desenvolvimento de deformidades e limitações nas atividades diárias. Sua etiologia é desconhecida, porém sugere-se que fatores ambientais, genéticos e infecções virais e bacterianas contribuem para o desencadeamento da doença. Sua incidência é maior em mulheres do que em homens e aumenta com a idade (Sociedade Brasileira de Reumatologia, 2011).

Segundo o Protocolo Clínico e Diretrizes Terapêuticas da Artrite (2019), para se chegar ao diagnóstico clínico de artrite reumatóide é necessário analisar os sinais e sintomas apresentados pelo paciente, exames laboratoriais e alterações em exames de imagens, como a radiografia, ressonância magnética e ultrassonografia, devendo considerar o tempo de evolução da doença, a determinação de autoanticorpos e avaliação da atividade inflamatória. Vale ressaltar que nenhum exame de qualquer natureza é eficiente isoladamente para comprovação do diagnóstico de artrite reumatóide. Este Protocolo também indica como critérios de classificação do American College of Rheumatology - ACR 1987 e ACR/European League Against Rheumatism ACR/EULAR 2010 para auxiliar no diagnóstico de A.R. Destaca-se ainda que, outros tipos de inflamações articulares devem ser consideradas no diagnóstico diferencial, como a artrite reativa, espondilite anquilosante, artrite psoriásica, lúpus eritematoso sistêmico e artrites associadas a doenças gastrointestinais, sendo que na fase erosiva, a osteoartrose também pode ser confundida no diagnóstico (Fuller, 2007).

Os sintomas iniciais da doença aparecem devido a tendinites, bursites e artrites que atingem inicialmente uma ou mais das pequenas articulações das mãos e dos pés de forma simétrica. Conforme a doença se estabelece, outras articulações podem ser acometidas, como as metacarpofalangeanas, interfalangeanas proximais e distais, atlantoaxial e temporomandibular. No entanto, tornozelo e regiões medianas do tarso e grandes articulações, como joelhos e ombros, geralmente são afetadas no estágio crônico da doença. O desvio ulnar, resultante da subluxação das articulações metacarpofalangeanas e da falange proximal da mão, e a tenossinovite do tendão flexor também são comuns na doença, gerando restrição de movimentos, de força, de mobilidade articular, dedos inseguros e queixas de rigidez matinal com melhora após atividade física. O paciente também pode apresentar fadiga, nódulos subcutâneos e pulmonares, vasculite reumatóide, anemia, depressão, febre, perda de peso e a síndrome de Sjogren secundária, caracterizada por olhos e boca secos (Fauci, 2014; Cartilha, 2011).

Para o tratamento da artrite reumatóide foram elaboradas recomendações pela Sociedade Brasileira de Reumatologia, tendo como base artigos baseados em evidências e opiniões de especialistas da Comissão de Artrite Reumatoide da SBR, por meio dos quais chegou-se a um consenso publicado em 2012, no qual sugere-se que o paciente e sua família devem ser elucidados sobre a doença e aborda as intervenções terapêuticas por meio de medicamentos, como anti-inflamatórios não hormonais (AINH), corticoides, drogas modificadoras do curso da doença (DMCD) e drogas imunodepressoras. Além disso, o Consenso evidencia as intervenções fisioterapêuticas, cirúrgicas, de terapia ocupacional e psicossocial no tratamento de AR.

Concomitante a SBR, o Conselho Federal de Fisioterapia e Terapia Ocupacional (COFFITO, 2013) recomenda como tratamento fisioterapêutico os exercícios de resistência para melhora da força muscular e do tempo de deslocamento, exercícios aeróbicos para melhora na qualidade de vida e capacidade funcional, ambos de maneira regular e uso de órteses de maneira individualizada. Para Wibelinger, 2015, os objetivos da fisioterapia no tratamento da A.R. são diminuir a atrofia, a fraqueza muscular e o edema, aumentar a flexibilidade, a amplitude de movimento, a resistência aeróbica e evitar posições viciosas. Porém, o COFFITO ressalta que há grande diversidade de técnicas fisioterapêuticas nos trabalhos disponíveis.

Portanto, com base nessas informações, a pesquisa propõe discutir as principais abordagens fisioterapêuticas disponibilizadas no meio de pesquisa científica para o tratamento de artrite reumatóide, tendo em vista a heterogeneidade de mecanismos disponíveis, tanto os comprovados cientificamente quanto os que necessitam de maiores esclarecimentos quanto a 
sua procedência na prática clínica e sua eficácia na evolução positiva do quadro álgico e funcional de pacientes que apresentam artrite reumatóide.

\section{Metodologia}

Segundo Marconi e Lakatos, 2003, a pesquisa bibliográfica trata-se de uma compilação dos principais trabalhos relevantes e atuais sobres o tema. O estudo de revisão da literatura permite um desenho de estudo indispensável de informações e um alinhamento sobre o tema. A habilidade do investigador e sua experiência permitirá que o material coletado seja uma descoberta de indícios e subsídios importantes para a pesquisa.

A pesquisa bibliográfica foi realizada nas bases de dados Cochrane, PEdro, Pubmed e Scielo. O trabalho limitou-se a revisões sistemáticas publicadas desde janeiro de 2010 até abril de 2021, tanto em língua inglesa quanto em portuguesa, nos quais foram excluídas revisões que abordavam sobre a fisiopatologia da artrite reumatóide, mas que não apresentavam estudos sobre o tratamento fisioterapêutico da doença. Também foram excluídos estudos que criam protocolos de elaboração de revisão sistemática sobre a A.R. Os descritores utilizados foram artrite reumatóide, fisioterapia, reabilitação, revisão sistemática. Durante a pesquisa foram encontrados 209 resultados, porém apenas 14 se encaixaram nos critérios de seleção escolhidos para elaboração desta revisão bibliográfica.

\section{Resultados e Discussão}

De acordo com o Quadro 1 podemos analisar 14 estudos que relatam principais abordagens fisioterapêuticas no tratamento de artrite reumatóide.

Quadro 1 - Resultado dos estudos publicados nos anos de 2010 à 2021.

\begin{tabular}{|c|c|c|}
\hline TÍTULO & AUTOR/ANO & RESULTADOS \\
\hline $\begin{array}{l}\text { Terapia por exercício para } \\
\text { artrite reumatóide: uma revisão } \\
\text { sistemática. }\end{array}$ & Küthe G.,2010 & $\begin{array}{l}\text { Nenhuma recomendação para terapia de treinamento pode ser feita } \\
\text { atualmente para pacientes com processo inflamatório ou destruição articular } \\
\text { significativa e radiologicamente detectável Altas cargas de exercício devem } \\
\text { ser evitadas em pacientes com lesões articulares. O papel do exercício físico } \\
\text { em pacientes com AR juvenil e pacientes com deficiências graves permanece } \\
\text { incerto. Em particular, em crises agudas e em idosos, pacientes gravemente } \\
\text { descondicionados, a fisioterapia convencional continuará sendo uma parte } \\
\text { essencial, pelo menos, do tratamento inicial. }\end{array}$ \\
\hline $\begin{array}{l}\text { Avaliação da qualidade das } \\
\text { diretrizes da prática clínica } \\
\text { sobre o uso de fisioterapia na } \\
\text { artrite reumatóide: uma revisão } \\
\text { sistemática. }\end{array}$ & $\begin{array}{l}\text { Hurkmans E. J. et. } \\
\text { al.,2011. }\end{array}$ & $\begin{array}{l}\text { Em geral, as recomendações das diretrizes sobre a intervenção } \\
\text { fisioterapêutica, tanto das diretrizes recomendadas quanto das não } \\
\text { recomendadas, careciam de detalhes quanto ao modo de liberação, } \\
\text { intensidade, frequência e duração. }\end{array}$ \\
\hline $\begin{array}{l}\text { Atividade física na artrite } \\
\text { reumatóide: Uma revisão } \\
\text { sistemática }\end{array}$ & $\begin{array}{c}\text { Tierney M. Et. al., } \\
2012\end{array}$ & $\begin{array}{l}\text { Os níveis de atividade física entre indivíduos com AR podem ser inferiores } \\
\text { aos recomendados e também quando comparados com controles saudáveis. } \\
\text { Esse achado ocorre independentemente da ferramenta de medida utilizada.. } \\
\text { Além disso, a necessidade de intervenção para melhorar os níveis de } \\
\text { atividade física na população com AR parece evidente com base nos achados } \\
\text { desta revisão. Os indivíduos com AR apresentam um risco excessivo de } \\
\text { doença cardiovascular em comparação com a população em geral, } 25,26 \text { e, } \\
\text { portanto, o incentivo à atividade física adequada parece fundamental e deve } \\
\text { ser uma prioridade para melhorar as taxas de mortalidade dessa população. }\end{array}$ \\
\hline $\begin{array}{l}\text { A eficácia da hidroterapia no } \\
\text { tratamento da artrite } \\
\text { reumatóide: uma revisão } \\
\text { sistemática. }\end{array}$ & $\begin{array}{c}\text { Al-Quebaeissy k. Y. } \\
\text { et. al. } 2012\end{array}$ & $\begin{array}{l}\text { Há algumas evidências que sugerem que a hidroterapia tem um papel } \\
\text { positivo na redução da dor e na melhoria do estado de saúde dos pacientes } \\
\text { com AR em comparação com nenhuma ou outras intervenções em curto } \\
\text { prazo. No entanto, o benefício a longo prazo é desconhecido. Mais estudos } \\
\text { são necessários. No entanto, os benefícios a longo prazo foram considerados } \\
\text { inconclusivos. }\end{array}$ \\
\hline
\end{tabular}




\begin{tabular}{|c|c|c|}
\hline $\begin{array}{l}\text { Uma revisão sistemática sobre } \\
\text { a eficácia da terapia com } \\
\text { exercícios manuais no } \\
\text { tratamento da artrite } \\
\text { reumatóide }\end{array}$ & $\begin{array}{l}\text { S.A. Bergstra et. al., } \\
2014\end{array}$ & $\begin{array}{c}\text { Pode-se concluir que a força de preensão e vários tipos de força de pinça } \\
\text { parecem ser melhorados pela terapia com exercícios manuais para pacientes } \\
\text { com AR, sem efeitos adversos na dor ou na atividade da doença. Apesar } \\
\text { desses efeitos positivos, deve-se ter cuidado ao tratar indivíduos com AR } \\
\text { ativa ou no período de exacerbação. }\end{array}$ \\
\hline $\begin{array}{l}\text { Balneoterapia (ou terapia de } \\
\text { spa) para artrite reumatóide }\end{array}$ & $\begin{array}{l}\text { Verhagen P.A. et al, } \\
2015\end{array}$ & $\begin{array}{c}\text { A evidência geral é insuficiente para mostrar que a balneoterapia é mais } \\
\text { eficaz do que nenhum tratamento, que um tipo de banho é mais eficaz do que } \\
\text { outro ou que um tipo de banho é mais eficaz do que pacotes de lama, } \\
\text { exercícios ou terapia de relaxamento. }\end{array}$ \\
\hline $\begin{array}{l}\text { A eficácia dos programas de } \\
\text { exercícios para as mãos em casa } \\
\text { na artrite reumatóide: uma } \\
\text { revisão sistemática }\end{array}$ & $\begin{array}{l}\text { Hammond A. e Prior } \\
\text { Y., } 2016\end{array}$ & $\begin{array}{l}\text { Os programas caseiros de exercícios para as mãos são eficazes para melhorar } \\
\text { a função da mão, a força de preensão e a dor na AR. Os programas de } \\
\text { exercícios de resistência de alta intensidade ensinados por terapeutas ao } \\
\text { longo de pelo menos várias sessões, incluindo estratégias para promover a } \\
\text { adesão a longo prazo, parecem ser mais eficazes e têm boa relação custo- } \\
\text { benefício. }\end{array}$ \\
\hline $\begin{array}{l}\text { Efeitos da reabilitação para o } \\
\text { alívio da dor em pacientes com } \\
\text { artrite reumatóide: uma revisão } \\
\text { sistemática }\end{array}$ & $\begin{array}{l}\text { Park Y, Chang } \\
\text { M. } 2016\end{array}$ & $\begin{array}{l}\text { A fisioterapia e a terapia ocupacional podem reduzir a dor na artrite } \\
\text { Reumatóide. A maioria dos estudos sugere que a Terapia Ocupacional e } \\
\text { Fisioterapia são mais benéficos do que os cuidados de reumatologia usuais. }\end{array}$ \\
\hline $\begin{array}{l}\text { Intervenção fisioterapêutica no } \\
\text { manejo de pacientes com artrite } \\
\text { reumatóide: uma revisão } \\
\text { sistemática }\end{array}$ & $\begin{array}{l}\text { Camacho-Bautista } \\
\text { K. et. Al., } 2016 .\end{array}$ & $\begin{array}{c}\text { A intervenção fisioterapêutica mais utilizada e que apresenta melhores efeitos } \\
\text { na população com Artrite Reumatóide é o exercício terapêutico moderado a } \\
\text { intenso, que deve ser prescrito tendo em conta o estado de saúde atual dos } \\
\text { pacientes. }\end{array}$ \\
\hline $\begin{array}{l}\text { Massagem terapêutica para dor } \\
\text { e função em pacientes com } \\
\text { artrite: uma revisão sistemática } \\
\text { de ensaios clínicos } \\
\text { randomizados }\end{array}$ & $\begin{array}{c}\text { Nelson N.L e } \\
\text { Churilla J.R, } 2017\end{array}$ & $\begin{array}{l}\text { Os resultados encontraram evidências de qualidade baixa a moderada de que } \\
\text { a massagem terapêutica é superior às terapias inativas na redução da dor e na } \\
\text { melhoria de certos resultados funcionais. Não está claro se a massagem } \\
\text { terapêutica é mais eficaz do que outras formas de tratamento. }\end{array}$ \\
\hline $\begin{array}{l}\text { Exercício para artrite } \\
\text { reumatóide da mão (revisão) }\end{array}$ & $\begin{array}{l}\text { W. Williams et. Al., } \\
2018\end{array}$ & $\begin{array}{c}\text { É incerto se o exercício melhora a função da mão ou a dor a curto prazo. } \\
\text { Provavelmente melhora ligeiramente a função, mas tem pouca ou nenhuma } \\
\text { diferença na dor a médio e longo prazo. É incerto se o exercício melhora a } \\
\text { força de preensão e pinça no curto prazo e provavelmente tem pouca ou } \\
\text { nenhuma diferença no médio e longo prazo. }\end{array}$ \\
\hline $\begin{array}{l}\text { Terapia Laser de Baixa } \\
\text { Intensidade para a redução da } \\
\text { dor em artrite reumatóide e } \\
\text { osteoartrite: uma revisão } \\
\text { sistemática. }\end{array}$ & $\begin{array}{l}\text { Fangel R. et. al. } \\
\qquad 2019\end{array}$ & $\begin{array}{l}\text { Existem evidências favoráveis de que a o laser de baixa intensidade pode } \\
\text { reduzir a dor em pacientes com osteoartrite de joelho, principalmente com } \\
\text { doses de } 6 \mathrm{~J} \text { por ponto e } 48 \mathrm{~J} \text { por sessão de tratamento. }\end{array}$ \\
\hline $\begin{array}{l}\text { O impacto da atividade física } \\
\text { nos níveis sericos de } \\
\text { marcadores inflamatórios na } \\
\text { artrite reumatóide: uma revisão } \\
\text { sistemátia da literatura. }\end{array}$ & $\begin{array}{l}\text { Burghardt R. et. al., } \\
2019\end{array}$ & $\begin{array}{l}\text { Esta revisão complementar revela que, até o momento, não há evidências } \\
\text { claras para apoiar um efeito independente mensurável de atividade física } \\
\text { sobre a concentração sistêmica de marcadores inflamatórios em pacientes } \\
\text { com AR. }\end{array}$ \\
\hline $\begin{array}{l}\text { Uma revisão sistemática que } \\
\text { explora as evidências relatadas } \\
\text { para sustentar a dose de } \\
\text { exercício em ensaios clínicos } \\
\text { de artrite reumatóide. }\end{array}$ & $\begin{array}{l}\text { Boniface G. Et. al., } \\
2020\end{array}$ & $\begin{array}{l}\text { A maioria dos ensaios clínicos randomizados incluídos não relatou estudos- } \\
\text { piloto ou evidências para sustentar a dose de exercício. No geral, as } \\
\text { descobertas indicam que o desenvolvimento e teste da dose de exercício em } \\
\text { ensaios clínicos é uma área que deve ser melhorada. }\end{array}$ \\
\hline
\end{tabular}

Fonte: Pereira e Maia (2021).

De acordo com a Figura 1, podemos analisar que a maioria dos estudos publicados entre 2010 e 2021 sobre as abordagens fisioterapêuticas no tratamento de artrite reumatóide, discutem sobre os efeitos dos exercícios físicos, demonstrando a importância da prática desse método nas intervenções clínicas. 
Figura 1 - Gráfico apresentando os resultados do estudo.

\section{Resultados encontrados de cada abordagem}

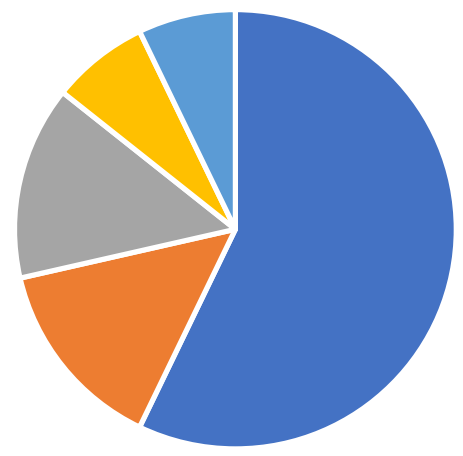

- Exercício físico " Hidroterapia = Laserterapia " Balneoterapia " Massagem terapêutica

Fonte: Pereira e Maia (2021).

\section{Exercícios físicos}

De acordo com Tierney et. al., 2012, pacientes com AR possuem elevados riscos de apresentarem doenças cardiovasculares, sendo a atividade física uma intervenção positiva essencial para a redução da taxa de mortalidade nesses indivíduos. Soma-se a isso, o relato de Burghardt et. al., 2019, no qual a atividade física proporciona benefícios na função cardiorrespiratória, força muscular, flexibilidade e desempenho neuromotor. Portanto, a atividade física é um fator determinante na melhora do quadro clínico do paciente com artrite reumatóide. No entanto, ao longo desta revisão, foram encontradas algumas discordâncias quanto a real eficácia dos exercícios terapêuticos e outras intervenções fisioterapêuticas, principalmente no que se refere aos parâmetros e dosimetrias dos tratamentos.

Boniface et. al., 2020, concluiu por meio de suas pesquisas que a maioria das evidências encontradas são insuficientes para determinar a dosimetria adequada para realização dos exercícios e não possui consenso quanto à qualidade, consistência e aplicabilidade das atividades, concomitante ao estudo de Hurkmnas et. al., 2011, no qual sugeriu que a intervenção fisioterapêutica necessita de evidências que descrevam quando a autorização do exercício que pode ser feita e qual seus parâmetros de intensidade, frequência e duração a serem recomendados aos pacientes com artrite reumatóide. Não obstante, o estudo sistemático de Camacho-Bautista et. Al., 2016, revelou que a intensidade de exercício mais adequada para o tratamento para pacientes com artrite reumatóide é a de nível moderado a intenso, o qual deve ser recomendado levando em consideração o quadro clínico atual do paciente, porém é de grande relevância que sejam feitas investigações de recomendações mais precisas.

Vale ressaltar que não há comprovações evidentes de quais os efeitos clínicos proporcionados pela atividade física nos biomarcadores inflamatórios de pacientes com artrite reumatóide, sendo necessária a realização de estudos randomizados sobre esses biomarcadores para comprovação dos efeitos dos exercícios (Burghardt et. al., 2019). Segundo Kuthe 2010, não são indicadas terapias de exercícios em pacientes que apresentam processo inflamatório ou alteração articular detectável radiologicamente devido à possibilidade de efeitos indesejáveis, devendo ser evitado exercícios de maior intensidade em pacientes com lesões articulares. Portanto, apesar de os exercícios físicos apresentarem efeitos positivos quanto à força de preensão, de pinça e ausência de efeitos adversos no quadro da doença, Bergstra et. al., 2014, alerta para o cuidado com paciente com artrite reumatóide ativa e em exacerbação. 


\section{Exercícios físicos para as mãos}

Segundo W. Williams et. Al., 2018, não é possível precisar a eficiência do exercício terapêutico na funcionalidade manual, no quadro álgico, força de preensão e pinça em curto período. Há, também, discreta evolução a médio e longo prazo, porém não há efeitos prejudiciais. Por outro lado, no estudo de Hammond e Prior, 2016 os exercícios para as mãos demonstram eficiência na melhora funcional da mão, na força de preensão e no quadro álgico de pacientes com artrite reumatóide, além disso, concluem que os exercícios de resistência de alta intensidade prescritos por terapeutas no decorrer do tratamento associados a estratégias de promoção da adesão por um longo período demonstraram maior eficácia, incluindo relação custo benefício e para prolongar este tratamento, faz-se necessário a conscientização de pacientes com artrite reumatóide na mão em relação à continuidade dos exercícios prescritos, propondo a criação de aplicativos educativos que demonstrem os exercícios e monitoram a evolução e qualidade que os exercícios estão sendo feitos pelo paciente.

\section{Massagem terapêutica}

Apesar de o exercício terapêutico ser o foco dos estudos sobre tratamento de artrite reumatóide, outras abordagens fisioterapêuticas são utilizadas. Como no estudo feito por Nelson e Churilla, 2017, no qual concluiu-se que por meio de evidências de nível baixo a moderado de estudos randomizados que a massagem terapêutica surte efeitos positivos no alívio da dor e funcionalidade de pacientes com artrite reumatóide.

\section{Hidroterapia}

Além desse recurso fisioterapêutico, a hidroterapia também se mostrou relevante no tratamento da artrite reumatóide com base no relato de Al-Quebaeissy et. al. 2012, no qual sugeriu que, em curto prazo, este método proporciona redução da dor e melhora na qualidade de vida de pacientes com artrite reumatóide. Os efeitos a longo prazo foram considerados inconclusivos nesse estudo.

\section{Laserterapia}

Do mesmo modo, a terapia com laser de baixa intensidade com aplicação de doses de $6 \mathrm{~J}$ por ponto e $48 \mathrm{~J}$ por sessão, ou seja, em 8 pontos diferentes por sessão, apresentou resultados positivos no quadro álgico de pacientes com artrite reumatóide e osteoartrite em joelho, porém o autor sugere que sejam feitos mais estudos sobre a dosimetria do laser em outras articulações afetadas pela doença (Fangel et. al. 2019).

\section{Conclusão}

As abordagens fisioterapêuticas em hidroterapia, laserterapia, massagem terapêutica e terapia com exercícios físicos e manuais apresentaram resultados positivos em relação a melhoria do quadro álgico e de saúde dos pacientes com artrite reumatóide por um curto período. Porém não há evidências suficientes que comprovem seus efeitos a médio e a longo prazo. Além disso, não há consenso quanto à dosimetria, intensidade, frequência e duração das abordagens terapêuticas, principalmente no que se refere aos exercícios físicos. Recomenda-se novos estudos que analisem os efeitos específicos das abordagens fisioterapêuticas e suas dosagens adequadas na artrite reumatoide, contribuindo assim para estudos futuros, uma vez que ainda existem muitas lacunas quanto aos parâmetros dos métodos.

\section{Referências}

Al-Qubaeissy K. Y. et. al. (2013). The effectiveness of hydrotherapy in the management of rheumatoid arthritis: a systematic review. Musculoskeletal Care. 11(1):3-18. 10.1002/msc.1028. 
Research, Society and Development, v. 10, n. 12, e439101220846, 2021

(CC BY 4.0) | ISSN 2525-3409 | DOI: http://dx.doi.org/10.33448/rsd-v10i12.20846

Bergstra S. A. et. al. (2014). A systematic review into the effectiveness of hand exercise therapy in the treatment of rheumatoid arthritis. Clin Rheumatol, 33(11):1539-48. 10.1007/s10067-014-2691-2.

Boniface G. et. al. (2020). A systematic review exploring the evidence reported to underpin exercise dose in clinical trials of rheumatoid arthritis. Rheumatology (Oxford), 59(11):p.3147-3157. 10.1093/rheumatology/keaa150.

Burghardt R. D. et. al. (2019). The impact of physical activity on serum levels of inflammatory markers in rheumatoid arthritis: a systematic literature review. Rheumatol Int, 39(5):p.793-804. 10.1007/s00296-019-04284-x.

COFFITO. (2013). Portaria $\mathrm{n}^{\circ}$ 710, de 27 de junho de 2013. Aprova o Protocolo Clínico e Diretrizes Terapêuticas da Artrite Reumatoide. https://www.coffito.gov.br/nsite/?s=artrite.

Fangel, R. et al. (2019). Low level laser therapy for reducing pain in rheumatoid arthritis and osteoarthritis: a systematic review. Fisioterapia em Movimento, v. 32, e003229. https://doi.org/10.1590/1980-5918.032.ao29.

Fauci, A. et. al. (2014). Reumatologia de Harrison. (3a ed.), AMGH.

Fuller R. (2007). Manual de reumatologia para graduação em medicina (2a ed.), Pontes.

Hammond A. \& Prior Y. (2016). The effectiveness of home hand exercise programmes in rheumatoid arthritis: a systematic review. Br Med Bull, 119(1):49-62. $10.1093 / \mathrm{bmb} / \mathrm{ldw} 024$.

Hurkmans E. J. et. al. (2011). Quality appraisal of clinical practice guidelines on the use of physiotherapy in rheumatoid arthritis: a systematic review. Rheumatology (Oxford), 50(10):1879-88. 10.1093/rheumatology/ker195.

Marconi M. A. \& Lakatos E. M. (2003). Fundamento de Metodologia Científica. (5a ed.), Atlas.

Ministério da saúde. (2019). Portaria conjunta nº 16, de 05 de novembro de 2019. Aprova o Protocolo Clínico e Diretrizes Terapêuticas da Artrite Reumatoide https://portalarquivos2.saude.gov.br/images/pdf/2019/novembro/08/SITE-Portaria-Conjunta-PCDT-Artrite-Reumatoide.pdf.

Mota, L. M. H et al. (2012). Consenso 2012 da Sociedade Brasileira de Reumatologia para o tratamento da artrite reumatoide. Rev. Bras. Reumatol., 52, 152174. http://dx.doi.org/10.1590/S0482-50042012000200002.

Nelson N. L. \& Churilla J. R. (2017). Massage Therapy for Pain and Function in Patients With Arthritis: A Systematic Review of Randomized Controlled Trials. Am J Phys Med Rehabil, 96(9): 665-672. 10.1097/PHM.0000000000000712.

Prado, A. D. et al. (2018). Ultrasound and its clinical use in rheumatoid arthritis: where do we stand? Adv. Rheumatol., 58:19. https://doi.org/10.1186/s42358018-0023-y.

Sociedade Brasileira de Reumatologia. (2021). Artrite Reumatoide. Doença inflamatória crônica que pode afetar várias articulações e com causa ainda desconhecida. https://www.reumatologia.org.br/doencas-reumaticas/artrite-reumatoide.

Tierney M. et. al. (2012). Physical activity in rheumatoid arthritis: a systematic review. J Phys Act Health., 9(7): 1036-48. 10.1123/jpah.9.7.1036.

Verhagen A. P. et. al. (2015). Balneotherapy (or spa therapy) for rheumatoid arthritis. Cochrane Library, 11;2015(4):CD000518. 10.1002/14651858.CD000518.pub2.

Wibelinger L. M. (2015). Fisioterapia em Reumatologia. (2a ed.). Revinter.

Williams M. A. et. al. (2018) Exercise for rheumatoid arthritis of the hand. Cochrane Library;7(7):CD003832. 10.1002/14651858.CD003832.pub3. 